\title{
OFDM SIGNAL ENERGY CHARACTERISTICS RESEARCH IN CHANNEL WITH PERMANENT PARAMETERS
}

\author{
Leonid O. Uryvskyi, Serhii O. Osypchuk
}

The Research Institute of Telecommunications, NTUU “KPI”, Kyiv, Ukraine

The simulation model for OFDM (Orthogonal Frequency Division Multiplexing) signal energy characteristics research is developed. The OFDM signal vector-phase states are examined. The momentary amplitudes distribution function is obtained. A hypothesis of amplitude distribution law is verified by the $\mathrm{X}^{2}$ criterion. The OFDM subcarriers optimal gain method is proposed.

\section{Introduction}

The OFDM technology is very popular in up-todate wireless telecommunication systems: $\mathrm{WiFi}$, WiMAX, LTE etc. [1,2]. The main target of such wide OFDM applications is a struggle against multipath propagation that occurs in places with meaningful objects which have an influence on radio propagation. The actual problem is the efficiency estimation of energy resources usage by OFDM technology in wireless channels with permanent and varying parameters. The channel with permanent parameters is examined in this work.

\section{Problem statement}

The purpose of this work is a research of OFDM signals energy characteristics. The object of research is energy resources efficiency estimation in OFDM technology. The subject of the study is optimal OFDM subcarriers amplification coefficient in the channel with permanent parameters.

Input parameters for research are the following: the number of subcarriers $N$, frequency band $\Delta F$, carrier frequency $F_{0}$ (all the above are frequency resources, frequency division); the amplifier linear section amplitude $U_{0}$, the type of subcarriers manipulation $M_{\mathrm{QAM}}=2$ for $\mathrm{QPSK}, M_{\mathrm{QAM}}=4$ for $\mathrm{QAM}-16$, $M_{\mathrm{QAM}}=6$ for QAM-64 (all the above are energy resources, amplitude division); the number and length of OFDM symbols (time resources, time division); required bit error probability on the receiving side $p_{\text {bit_req }} \quad$ (reliability requirement for received information).

The following tasks were set to evaluate the efficiency usage of the energy resources by OFDM in wireless channels:
- develop a simulation model in the MatLab software for OFDM signals energy characteristics research;

- build up an OFDM subcarriers vector gain-phase diagram in discrete points of time;

- extract the momentary OFDM signal amplitudes distribution function;

- identify the OFDM signal amplitudes distribution function law;

- test a distribution function law hypothesis of OFDM signal amplitudes by $\mathrm{X}^{2}$ criterion;

- determine an optimal OFDM subcarriers gain coefficient $K_{P_{-} \text {opt }}$ per the following criterion: an equality of i-subcarrier symbol error probability $p_{i_{-} s y m b}$ and probability of amplifier non-linear distortions $p_{N L}$ with subcarriers amplification and permanent channel parameters.

\section{OFDM signal}

The OFDM subcarriers representation is shown on the fig. 1.

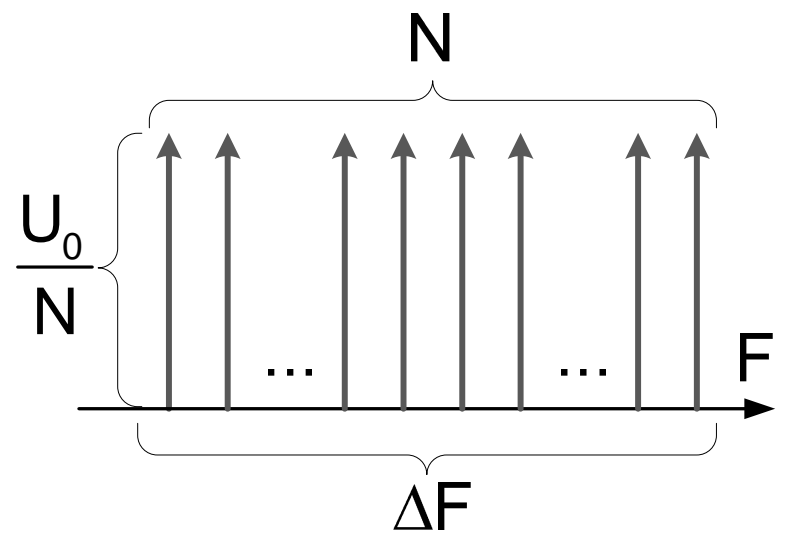

Fig.1. OFDM subcarriers representation

OFDM signal consists of $N$ orthogonal subcarriers. OFDM subcarriers are modulated by $N$ parallel data flows. The frequency independence is provided by 
mutual subcarriers orthogonality on the symbol length [1]:

$$
\int_{0}^{T_{S}} \sin 2 \pi f_{n} t \cdot \sin 2 \pi f_{n} k=0, n \neq k
$$

\section{Single carrier transmission: channel parameters and signal energy detection}

The simulation model for OFDM signal research is developed in MatLab software. A computation of white noise level $\mathrm{N}_{0}$ is based on the following input parameters: $U_{0}, \Delta F, M_{Q A M}$. All the above parameters are used to reach the required bit error probability $p_{\text {bit_req }}$ at single carrier transmission in the channel with a specific noise value $\mathrm{N}_{0}$. A signal-noise ratio is calculated as

$$
h_{0}^{2}=\frac{E_{c}}{\mathrm{~N}_{0}}=\frac{U_{0}^{2} T_{0}}{\mathrm{~N}_{0}},
$$

where $T_{0}=1 / \Delta F$, i.e. for the case of transmission based on single carrier and using a full range of amplifier amplitude linear section.

The bit error probability $p_{b i t_{-} 0}$ and symbol error probability $p_{\text {symb_0 }}$ for different types of modulation are calculated by well-known formulas for QPSK and QAM modulations [3]:

$$
\begin{aligned}
& p_{\text {symb_QPSK } \_0}\left(h_{0}^{2}\right)=\frac{3}{4}-\frac{1}{2} \Phi\left(\sqrt{h_{0}^{2}}\right)-\frac{1}{4} \Phi^{2}\left(\sqrt{h_{0}^{2}}\right) ; \\
& p_{\text {bit_QPSK_0 }}\left(h_{0}^{2}\right)=\frac{1}{2}\left[1-\Phi\left(\sqrt{2 h_{0}^{2}} \cos \frac{\pi}{4}\right)\right],
\end{aligned}
$$

where $\Phi(x)=\frac{2}{\sqrt{2 \pi}} \int_{0}^{x} e^{-\frac{t^{2}}{2}} d t-$ the Kramp function, $x=\sqrt{h_{0}^{2}} ;$

$$
\begin{gathered}
p_{\text {symb_QAM_0 }}\left(h_{0}^{2}\right)=1-\left(1-\frac{2\left(1-\frac{1}{\sqrt{M}}\right)}{\sqrt{2 \pi}} \int_{\sqrt{\frac{3 k}{M-1} h_{0}^{2}}}^{\infty} \exp \left(\frac{-u^{2}}{2}\right) d u\right)^{2} \\
p_{\text {bit_QAM_0 }}\left(h_{0}^{2}\right)=\frac{4\left(1-\frac{1}{\sqrt{M}}\right)}{k \sqrt{2 \pi}} \int_{\sqrt{\frac{3 k}{M-1} h_{0}^{2}}}^{\infty} \exp \left(\frac{-u^{2}}{2}\right) d u,
\end{gathered}
$$

Thereby, the white noise value $N_{0}$ is calculated for the case when the bit error probability $p_{b i t_{-} 0}$ is equal to required bit error $p_{\text {bit_req }}$ :

$$
N_{0}=f\left(h_{0}^{2}=f\left(U_{0}, \Delta F, M\right)\right), p_{\text {bit_o }}=p_{\text {bit_req }}
$$

The above expression may be written in other way:

$$
U_{0}=f\left(h_{0}^{2}=f\left(N_{0}, \Delta F, M\right)\right), p_{\text {bit_ } 0}=p_{\text {bit_req }}
$$

i.e. searching the required value of amplifier linear amplitude part $U_{0}$ to satisfy the condition $p_{\text {bit_o }}=p_{\text {bit_req }}$ and specific signal to noise ratio achievement with the known values of white noise value, modulation type and frequency band.

The random digits generator is used in MatLab system for modeling the pseudorandom information bits sequence. Verification the random digits generator on series condition shows an average value $\sim 0.5$ (the ratio of digits that satisfied a check on series to cumulative value of generated digits), and it characterizes the random digits generator in MatLab as enough good to generate a pseudorandom information bits sequence.

As was shown, three manipulation types are realized in simulation model: QPSK, QAM-16 and QAM-64. Each subcarrier of OFDM symbol matches to the appropriate initial amplitude and phase. It occurs according to the selected subcarriers manipulation type and information bits.

\section{Momentary OFDM signal values}

The momentary OFDM signal values $U_{\text {OFDM_t }_{i}}(t)$ are calculated with the step 1/360 of maximal subcarrier frequency value $f+\Delta F(N-1) / 2 N$ :

$$
U_{\text {OFDM }_{-} t_{i}}(t)=\sum_{n=-N / 2}^{N / 2} U_{n} \cdot \sin \left(2 \pi f n t+\varphi_{n}\right),
$$

where $U_{n}=U_{0} / N$ is a subcarrier amplitude, $f$ subcarrier frequency, $n$ - subcarrier number, $\varphi_{n}-$ initial phase.

Some gain-phase vector diagrams are shown on the figures below. Each vector demonstrates a graphical presentation of subcarrier gain and subcarrier phase instant state. The vector length is an amplitude value, and the angle position is an initial phase. The following is shown on the graphs below:

1. The separated positions of vectors are demonstrated on the fig.2,a,1 and fig.2,b,1. Blue vectors lie on the left of frequency band center and have a smaller frequency values than carrier frequency. Black vectors lie on the right of frequency band center and have a bigger frequency values than carrier frequency.

2 . The position of vectors one by one is shown on the fig.2,a,2 and fig.2,b,2. It means the end of the first vector is start of the second vector and so on. The red vector on fig.2,a, 2 and fig.2,b,2 shows the summary vector that is the result of vectors composition. The red 
vector defines an amplitude and phase of OFDM signal in some specific point of time.

3. Momentary OFDM signal values in time domain are shown on the fig.2,a,3 and fig.2,b,3.

The OFDM subcarrier vectors position is shown on fig. 2,a,b in points of time $t_{0}=0$ and $t_{1}=T_{0} / 12$. The 8 OFDM subcarriers are demonstrated for a better clearness. Each OFDM subcarrier is modulated by the signal mapping QAM-16, i.e. 4 bits per symbol. All the subcarriers are modulated by the same symbols " 1111 " for better look-and-feel. The symbol "1111" defines the same initial amplitude and phase for each subcarrier. All the vectors have the same amplitude " 1 " and the same initial phase $45^{\circ}$ as shown on the fig. 2,a,1.

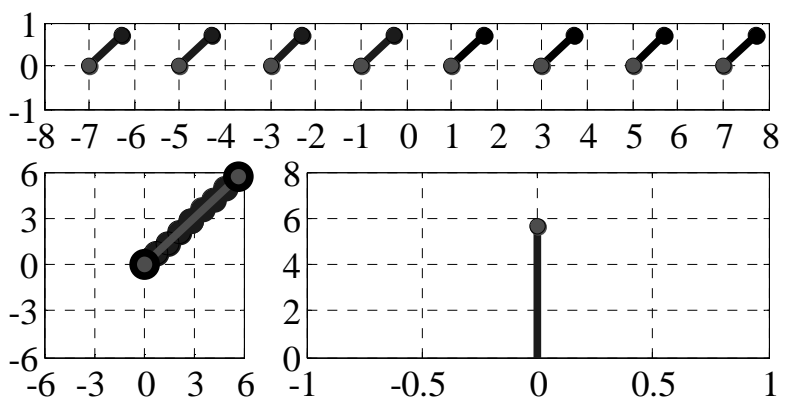

a)
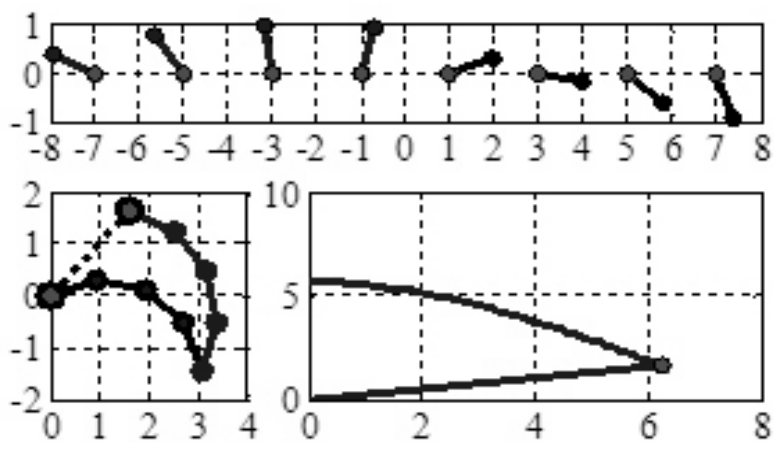

b)

Fig.2. OFDM subcarriers vector-phase diagram and amplitude behavior in points of time

$$
t_{0}=0 \text { and } t_{1}=T_{0} / 12
$$

\section{OFDM signal PAPR}

PAPR (Peak to Average Power Ratio) [4,5] is a ratio of maximal signal power $P_{\max }$ to average signal power $P_{\text {avg }}$ per some period of time:

$$
\mathrm{PAPR}_{\mathrm{OFDM}}=\frac{P_{\max }}{P_{\text {avg }}},
$$

where $P_{\max }=U_{i_{\max }}^{2} ;$ average amplitude values: $P_{\text {avg }}=\frac{1}{j} \sum_{i=1}^{j} U_{i}^{2}$, where $j$ - the number of amplitudes values.

It is known that OFDM signal has a big PAPR value. A bigger OFDM subcarriers number brings to a bigger PAPR value. This is an imperfection of OFDM technology; it leads to inefficient energy resources usage. Some methods are proposed and implemented for struggle against high PAPR, such as bits interleaving, clipping etc. These methods allow to reach a smaller PAPR values.

\section{OFDM signal momentary amplitudes distribution function research}

The OFDM momentary amplitudes distribution function is based on statistic information of OFDM signal levels. The OFDM amplitudes distribution function is shown on fig. 3 for specific OFDM parameters $N, U_{0}, \Delta F, M_{\mathrm{QAM}}$.

As shown on the fig.3, the subcarriers number growing and modulation type increasing more and more result in the normal distribution law of OFDM momentary amplitude values:

$$
f(x)=\frac{1}{\sigma \sqrt{2 \pi}} e^{-\frac{(x-\mu)^{2}}{2 \sigma^{2}}},
$$

where $\mu$ is a mathematical expectation of distribution and $\sigma$ is a dispersion of distribution. It's obvious that a mathematical expectation is equal to zero in the case of OFDM amplitudes distribution: $\mu=0$. The OFDM amplitudes values are represented by modulo on the fig.3, and it means that there are double values on ordinate axis. As the research has shown, the dispersion of distribution is a function of the OFDM subcarriers number: $\sigma=f(N)$. The dispersion and subcarriers number dependency is shown on the fig.4.

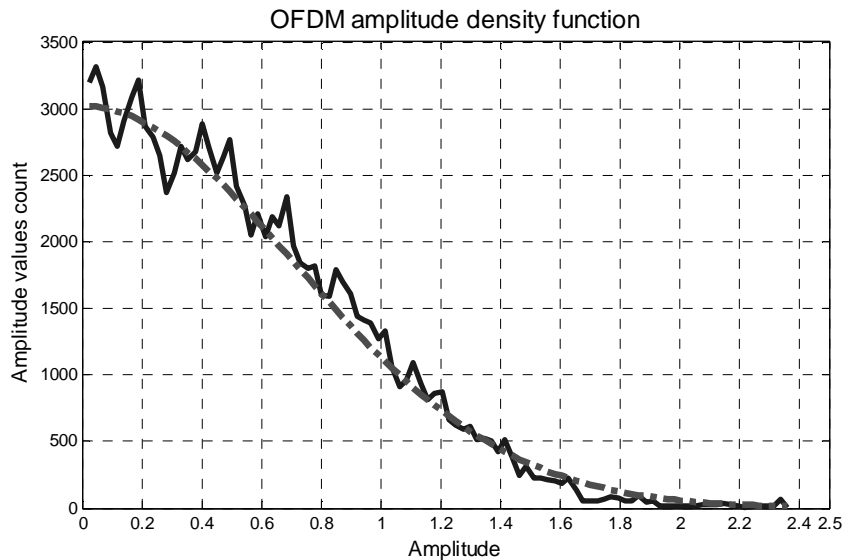

a) 


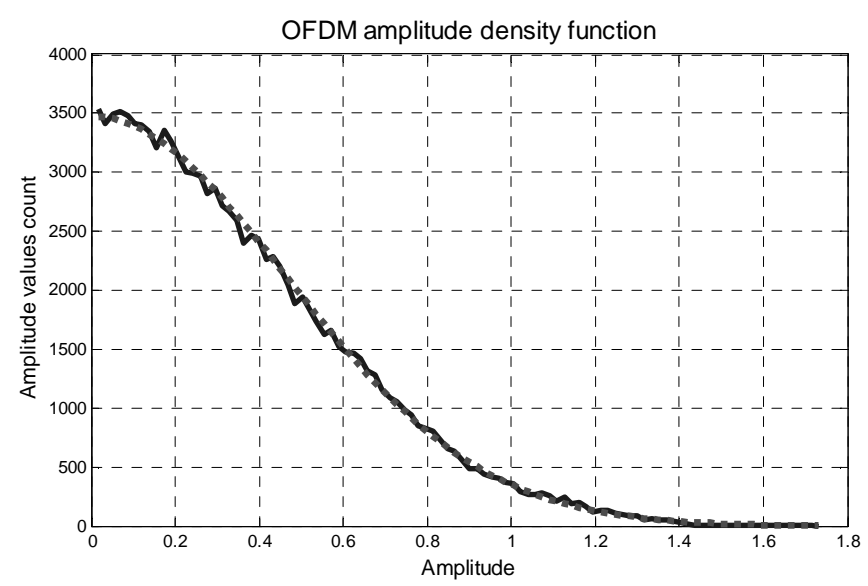

b)

Fig.3. OFDM amplitudes distribution functions obey to normal distribution law:

a) $\mathrm{N}=16$, QAM-16; b) $\mathrm{N}=32$, QAM-64

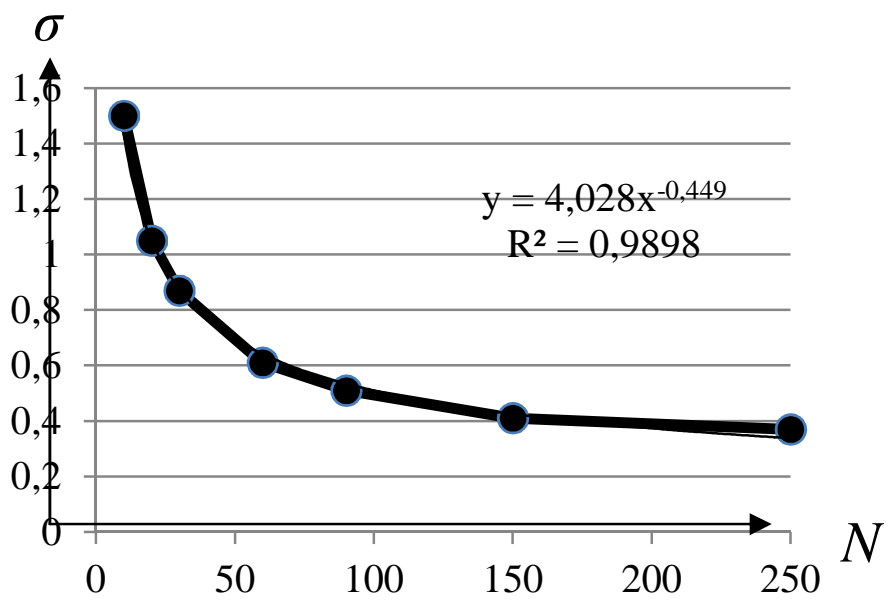

Fig.4. The dispersion and subcarriers number dependency: $\sigma(N)=4.028 \cdot N^{-0.44}$,

the approximation reliability is 0.989

The OFDM amplitudes normal distribution law hypothesis is confirmed by the $\mathrm{X}^{2}$ criterion.

\section{Analytic description of OFDM energy model}

It is well-known, that OFDM PAPR values are big and these are growing proportionally to the OFDM subcarriers number. It means that a maximal OFDM amplitude value is significantly bigger, than an average OFDM amplitude value over a specific period of time. A maximal OFDM amplitude value can be reached when subcarriers' amplitudes are maximal and equal; moreover, subcarriers initial phases are equal too. This case on a vector-phase diagram corresponds to the vectors position with the same length and initial phase of subcarriers; but this is very unlikely case, and an average OFDM signal amplitude values is considerably lesser than maximal amplitude value. It means that an average OFDM amplitude value is meaningfully smaller than a linear part of power amplifier characteristic. In connection with that it stands to be possible to gain the OFDM subcarriers level and do not exceed the linear part of power amplifier characteristic and prevent the non-linear distortions. In this case it is possible to talk about the optimal subcarriers gain coefficient $K_{U_{-} o p t}$. The optimality criterion meets the one of two positions:

1) OFDM subcarriers optimal gain coefficient when non-linear distortions appear with certain acceptable probability value, i.e. exceeding the linear part of power amplifier characteristic by OFDM signal value;

2) OFDM subcarriers optimal gain coefficient when the probability of non-linear distortions is equal to symbol error probability on OFDM subcarrier: $p_{N L_{-} \text {dist }}=p_{\text {symb } i}$.

In the first case the probability of exceeding the power amplifier linear part characteristic is equal to zero, because each subcarrier has only a part $U_{i}=U_{0} / N$ of total linear part voltage $U_{0}$, where $N$ is the number of OFDM subcarriers. Due to big PAPR value it is reasonable to amplify the OFDM subcarriers level up to the point when an exceeding the power amplifier linear part characteristic happens and nonlinear distortions appear.

So far as receiver works with a symbols but not with a bits, in the second case the symbol error probability is reviewed for each OFDM subcarrier to meet the following expectation: $p_{N L_{-} \text {dist }}=p_{\text {symb }_{-} i}$. Let's review the energy parameter $h_{0}^{2}$ firstly that shows the signal element energy $E_{c}=P_{c} T_{0}$ and noise spectral concentration $\mathrm{N}_{0}$ ratio on the input part of receiver:

$$
h_{0}^{2}=\frac{P}{\mathrm{~N}_{0} V_{0}}=\frac{P_{c} T_{0}}{\mathrm{~N}_{0}}
$$

For $N$ OFDM subcarriers,

$$
U_{0}=\sum_{i=1}^{N} U_{i}=U_{i} \cdot N
$$

Whereas the power is proportional to square of voltage:

$$
P \sim U_{0}^{2}=\left(U_{i} \cdot N\right)^{2}=N^{2} \cdot U_{i}^{2}=N^{2} \cdot P_{i}
$$

It is shown in the above expression that power of one OFDM subcarrier is $N^{2}$ times smaller, than a power of single carrier transmission; the voltage of one OFDM subcarrier is $N$ times smaller, than a voltage of single carrier transmission:

$$
P_{i}=\frac{P_{0}}{N^{2}} ; U_{i}=\frac{U_{0}}{N}
$$


The channel speed $V_{i}$ of one OFDM subcarrier is $N$ times lesser comparatively with a single carrier transmission; the symbol length is $N$ times longer comparatively with a single carrier transmission:

$$
V_{i}=\frac{V_{0}}{N} ; T_{i}=T_{0} \cdot N
$$

The energy parameter for one OFDM subcarrier $h_{i}^{2}$ is $N$ times smaller, than energy parameter $h_{0}^{2}$ of single carrier transmission:

$$
h_{i}^{2}=\frac{P_{i}}{\mathrm{~N}_{0} V_{i}}=\frac{\frac{P}{N^{2}}}{\mathrm{~N}_{0} \frac{V_{0}}{N}}=\frac{P}{N \mathrm{~N}_{0} V_{0}}=\frac{h_{0}^{2}}{N}
$$

\section{The optimal OFDM subcarriers gain factor}

Assume that the requirement for bit error rate on a receiver side is $p_{b i t_{\text {req }}}=10^{-6}$. A symbol error probability is bigger, than a bit error probability. At the initial moment the power amplifier linear characteristic part is evenly distributed between all the OFDM subcarriers and the nonlinear distortions initiation probability is equal to zero: $U_{i}=\frac{U_{0}}{N} ; p_{N L_{-} \text {dist }}=0$. In this case the symbol error probability $p_{\text {symb_o }}$ is reviewed firstly on the receiver input so far as receiver handles a symbol, and it is possible to estimate actually the symbol error probability $p_{\text {symb_o }}$ in the first place. After decoding it is possible to evaluate the bit error probability $p_{b i t \_0}$. It's important to highlight that the bit error probability is smaller than the symbol error probability: $p_{\text {symb_0 }}>p_{\text {bit_0 }}$. In the result, the required criterion from a user is a specific bit error probability $p_{\text {bit_req }}$, but not a symbol error probability. So far as the energy parameter $h_{i}^{2}$ of each OFDM subcarrier in $N$ times lesser than $h_{0}^{2}$, then the below expression is obvious:

$$
p_{\text {symb } \_i} \gg p_{\text {symb_ } 0} \gg p_{N_{-} \text {dist }}
$$

If OFDM subcarriers level increases, then total subcarriers amplitude grows and numerically exceeds the initial power amplifier linear characteristic part $U_{0}$; the nonlinear distortions probability increases in this case:

$$
p_{N L_{-} \text {dist }} \uparrow=f\left(U_{i} \uparrow\right)
$$

Correspondingly, together with OFDM subcarriers gain, the symbol error probability decreases:

$$
p_{\text {symb_i }} \downarrow=f\left(U_{i} \uparrow\right)
$$

Thus, when initially we have $p_{\text {symb_i }} \gg p_{N_{L} \text { dist }_{1}}$, and if gain the subcarriers level $U_{i} \uparrow$, then the symbol error probability on each subcarrier will be decreasing $p_{\text {symb_i }} \downarrow$, and the nonlinear distortions probability will be growing $p_{N L_{-} \text {dist }} \uparrow$. So there will be a point when $p_{\text {symb } i}=p_{N_{-} \text {dist }}$. The optimal power and amplitude gain coefficients:

$$
\begin{aligned}
& U_{i_{-} o p t}= K_{U_{-} o p t_{-} i} \cdot \frac{U_{0}}{N}=K_{U_{-} o p t_{-} i} \cdot U_{i} \\
& P_{i_{-} o p t}= K_{P_{-} o p t_{-} i} \cdot \frac{P_{0}}{N^{2}}=K_{P_{-} o p t_{-} i} \cdot P_{i} \\
& K_{P_{-} o p t_{-} i}=K_{U_{-} o p t_{-} i}^{2}
\end{aligned}
$$

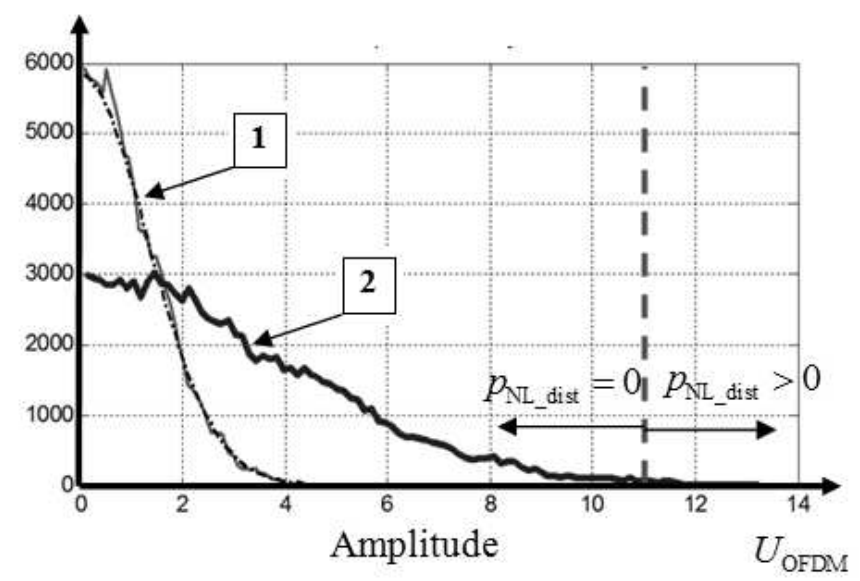

a)

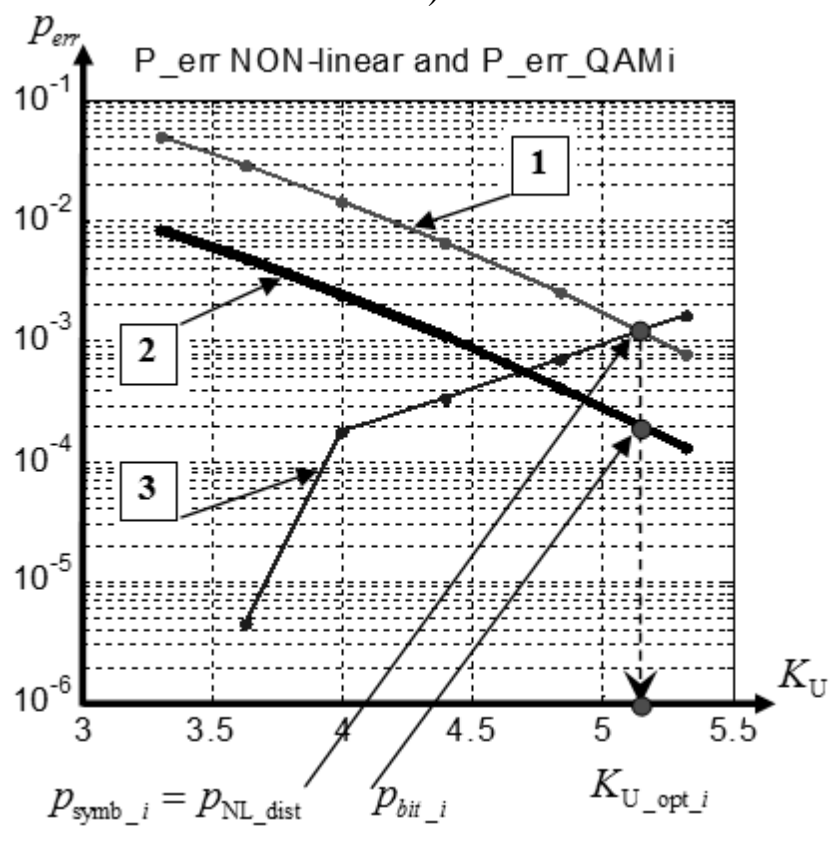

b)

Fig.5. The condition achievement: $p_{s y m b_{-} i}=p_{N L_{-} \text {dist }}=1,1 \cdot 10^{-3}$ when $K_{U_{-} o p t_{-} i}=4,83$; 
achieved BER: $p_{b i t_{-} i}=2 \cdot 10^{-4}$. OFDM subcarriers number: $N=10$, modulation type: QAM-64; achieved BER on single carrier transmission with usage of the same energy resources (required BER): $p_{\text {bit_req }}=10^{-6}$ ).

a) OFDM amplitudes distribution function;

$$
\text { b) } p_{\text {err }}=f\left(K_{U}\right)
$$

The OFDM amplitudes distribution function (the number of amplitudes as a function of amplitude values: $X_{m}=f\left(U_{m}\right)$, where $m$ is a specific point of time) is shown on the fig.4,a: 1 - the initial distribution function, before subcarriers gain; 2 - the results distribution function, after subcarriers gain with an amplifying coefficient $K_{U_{-} o p t_{-} i}$ and reaching the condition $\quad p_{s y m b} i=p_{N L_{-} \text {dist }}$. On the fig.5, $\mathrm{b}$ the dependencies of bit $p_{b i t}$, symbol $p_{\text {symb }}$ and non-linear distortion probabilities $p_{N L_{-} \text {dist }}$ of the subcarriers gain coefficient $K_{U}$ are shown. The fig.5,b shows dependencies for a specific set of OFDM subcarriers number and modulation type; varying these parameter will bring to different intersection point $K_{U_{-} o p t_{-} i}: p_{\text {symb_i }}=p_{N_{L_{-}} \text {dist }}$ :

$$
\begin{aligned}
& K_{U_{-} o p t_{-} i}=f\left(\left\{p_{s y m b_{-} i}, p_{N L_{-} d i s t}\right\}=f\left(N, k, K_{U}\right)\right), \\
& p_{s y m b_{-} i}=p_{N L_{-} d i s t}
\end{aligned}
$$

So, the bit error probability is reached $p_{b i t_{-} i}=2 \cdot 10^{-4}$ with OFDM when client's requirement is $p_{\text {bit_req }}=10^{-6}$. To reach the required BER level, it is needed to use some additional methods for reliability growth, such as antinoise coding [6].

\section{Conclusion}

The OFDM technology is always unprofitable with integrity and power resource effectiveness usage in comparison with single carrier transmission in the channel with the additive white gaussian channel.

The modeled vector-phase OFDM signal states and the given OFDM amplitude distribution functions have shown that an amplitude distribution function law approximates to the normal distribution law with specific characteristics against the OFDM signal parameters. This hypothesis is verified and confirmed by the $\mathrm{X}^{2}$ criterion.
The proposed method of OFDM signal usage supposes an obtaining and utilization of the optimal gain coefficient $K_{U_{-} \text {opt }}$ for OFDM subcarriers. The optimality criterion is an achievement of condition $p_{\text {symb } i}=p_{N L_{-} \text {dist }}$ against the subcarriers number $N$ and subcarriers modulation type.

A gain of OFDM subcarriers stops when the condition $p_{s y m b} i=p_{N L_{-} \text {dist }}$ is met. The required BER in this case is significantly lesser that reached BER value on one OFDM subcarrier:

$$
p_{\text {bit_req }}<<p_{\text {bit_ } i}<p_{\text {symb_i } i}=p_{N L_{-} \text {dist }}
$$

OFDM subcarriers amplification by the proposed method gives an opportunity to use OFDM technology on the limit of permissible and regulated power beaming. It provides better range and reliability for information transmission in comparison with OFDM technology usage without subcarriers gain.

To reach the required BER level, it is needed to use some additional methods for reliability growth, such as antinoise coding.

The described results are received in MatLab software; the simulation model for analysis of OFDM signal power resources usage is developed.

\section{References}

1. R. Prasad. OFDM for wireless communications systems. - Artech House Universal Personal Communications series, 2004. - $291 \mathrm{p}$.

2. H. Schulze, C.Leuders. Theory and Applications of OFDM and CDMA: Wideband Wireless Communications. John Wiley \& Sons Ltd, 2005. - 421 p.

3. Fink L. Discrete messages transmission theory. - M.: "Sov.Radio", 1970. - 728 p.

4. H. Ochiai and H. Imai, "On the Distribution of the PeaktoAverage Power Ratio in OFDM Signals," IEEE Trans. Commun., vol. 49, no. 2, pp. 282-89, Feb. 2001.

5. Seung H. Han, Jae H. Lee, "An overview of peak-toaverage power ratio reduction techniques for multicarrier transmission," IEEE Wireless Communications, vol.12, no. 2, pp. 56-65, 2005.

6. B. Lu, X. Wang, and K. R. Narayanan, BLDPC-based space-time coded OFDM systems over correlated fading channels: Performance analysis and receiver design, [in Proc. 2001 IEEE Int. Symp. Information Theory (ISIT '01), Jun. 24-29, 2001, vol. 1, p. 313.

Received in final form December 4, 2013 